\title{
EXCESS RF POWER REQUIRED FOR RF CONTROL OF THE SPALLATION NEUTRON SOURCE (SNS) LINAC, A PULSED HIGH- INTENSITY SUPERCONDUCTING PROTON ACCELERATOR *
}

\author{
Michael Lynch, Sung-Il Kwon, Amy Regan, Yi-Ming Wang, LANL, Los Alamos, NM 87545 USA
}

\section{Abstract}

A high-intensity proton linac, such as that being planned for the SNS, requires accurate RF control of cavity fields for the entire pulse in order to avoid beam spill. The current design requirement for the SNS is RF field stability within $\pm 0.5 \%$ and $\pm 0.5^{\circ}[1]$. This RF control capability is achieved by the control electronics using the excess RF power to correct disturbances. To minimize the initial capital costs, the RF system is designed with 'just enough' RF power. All the usual disturbances exist, such as beam noise, klystron/HVPS noise, coupler imperfections, transport losses, turn-on and turn-off transients, etc. As a superconducting linac, there are added disturbances of large magnitude, including Lorentz detuning and microphonics. The effects of these disturbances and the power required to correct them are estimated, and the result shows that the highest power systems in the SNS have just enough margin, with little or no excess margin.

\section{OVERVIEW OF SNS}

The SNS is a $1 \mathrm{GeV}$ linac, followed by an accumulator ring and neutron spallation target. The SNS is being built at Oak Ridge National Laboratory by a 6 laboratory collaboration. Los Alamos has responsibility for the room temperature portions of the linac, as well as the RF systems for the entire linac [2], [3]. SNS is very concerned with beam spill because of the resulting activation of the hardware that can come from the spilled beam. Numerous discussions between LANL, Brookhaven and ORNL lead to a specification for the cavity field stability of $0.5 \%$, $0.5^{\circ}$. Excess power capability leads to excess costs, and the entire collaboration has been very interested in minimizing the costs for the project construction. The decision was made to buy the minimum power level in the klystron that is needed in the highest power portion of the superconducting sections. That turned out to be $550 \mathrm{~kW}$. Follow-on changes and discussions by SNS engineers and physicists and by review committees questioned whether there was sufficient, or perhaps, too much excess margin. The questioning came from two directions. One was a concern that there was not enough margin, and the system would not function properly. The other is that there was too much margin, and the system could accelerate more beam, or power cavities at higher gradients. This paper summarizes the analysis that was done.

*Work supported by the Office of Basic Energy Science, Office of Science of the US Department of Energy, and by Oak Ridge National Laboratory.

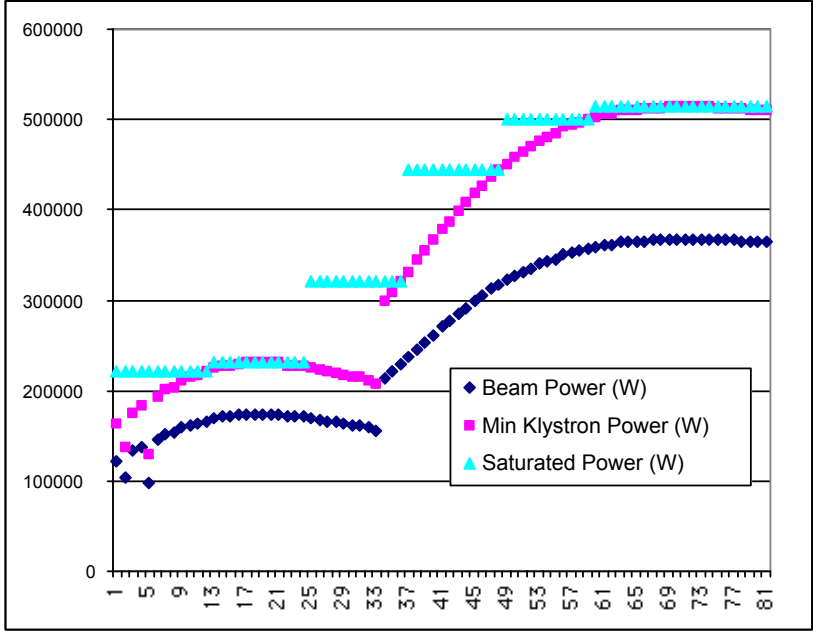

Figure 1: Klystron saturated power setting, beam power, and min. klystron power per superconducting module.

\section{RF POWER MARGIN}

Because of the configuration with multiple tubes on one power supply in the superconducting portion of the accelerator where as many as 12 klystrons are powered from a single $\mathrm{HV}$ source, only some of the klystrons operate with their minimum margin. Figure 1 shows the saturated power setting and the required power of each klystron. As can be seen in the figure, only some klystrons operate near the full rated power of $550 \mathrm{~kW}$ with the minimum excess power margin. The margin estimate is $33 \%$ for the low beta cavities, and $40 \%$ for the high beta cavities.

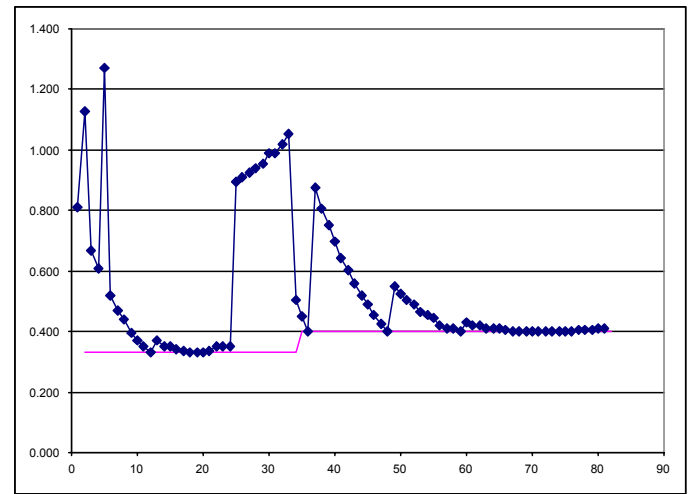

Figure 2: Lower line is the minimum excess power margin. Upper line is the actual margin per module. 
The curve in figure 1 can be viewed differently, as shown in figure 2 . In this case, the amount of margin above that estimated for the beam and cavity is shown for each RF module. Due to the combination of multiple klystrons on one supply (so that all klystrons on that supply operate with the same saturated power) and the fact that each module needs a different amount of power, most modules will have more than the minimal amount specified.

\subsection{Source of the Margin Requirements}

The margin requirements come from the following considerations: losses between the klystron and cavity (either from resistive losses or mismatches), errors in the cavity (frequency setting, Lorentz force detuning, Microphonics), errors in the waveguide-to-cavity coupler, errors in the beam (amplitude and/or synchronous phase), klystron performance/response (unit differences in gain and phase or changes due to $\mathrm{HV}$ variations), speed of response and accuracy of the feedback control system.

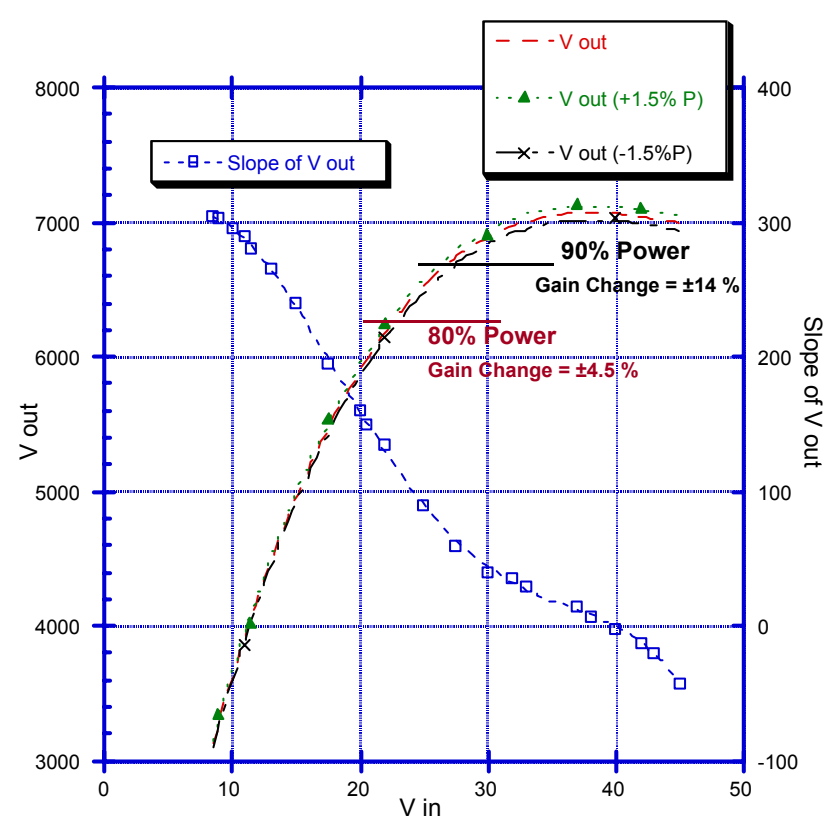

Figure 3. Klystron gain curve and slope of that curve. Also shown are the variations due to $1 \%$ Voltage changes.

The last item is directly related to the RF generator operating point. See Figure 3. The slope of the klystron gain curve represents the primary gain element for the cavity field control system. As the tube is operated near saturation, the gain approaches zero. At saturation, there is zero gain available for control of disturbances. This impact is felt even when operating near saturation. Figure 3 shows the effect of power supply variation on the feedback gain. A $1 \%$ variation in the $\mathrm{HV}$ (perhaps from ripple), has a much bigger effect on operation when the klystron is operating closer to saturation. At $90 \%$ of saturation, the gain changes $\pm 14 \%$ with a $\pm 1 \%$ change in
HV. When operating at $80 \%$ of saturation, the gain changes only $\pm 4.5 \%$ with the same $\mathrm{HV}$ variation.

\subsection{Feedforward}

There are some techniques to mitigate the control disturbances in addition to the use of feedback control. The primary method is the use of feedforward, which can be extremely effective on repetitive disturbances [4]. These include power supply ripple, steady state errors in the beam or cavity settings, etc.

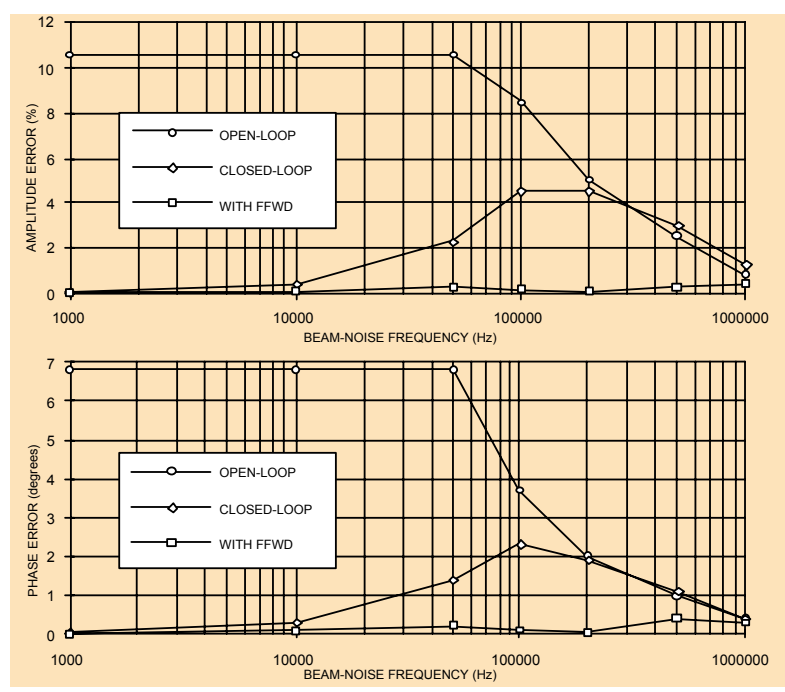

Figure 4. Errors open loop, with feedback, and with feedback and feedforward.

Figure 4 shows the effect graphically (from a system model). Steady state noise of various frequencies was introduced to a system. One curve shows the error in an open loop mode. One curve show the errors with feedback only. The lowest curve shows the result with feedforward applied. The dramatic effect on repetitive disturbances, both in error reduction and in bandwidth, is readily apparent.

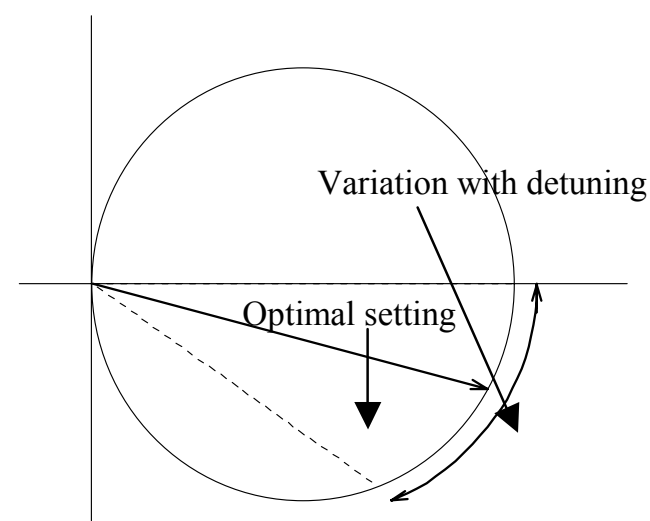

Figure 5. Response of cavity as the cavity is detuned. Optimal setting is the center vector.

\subsection{Lorentz Detuning and Microphonics}

For the high beta cavities, the expected Lorentz detuning effects during the pulse will be in the range of 
$300 \mathrm{~Hz}$. Based on that number, and a microphonics range of about $100 \mathrm{~Hz}$, the amplitude response will vary up to $+8,-12 \%$ And the phase response will vary by up to $+7,-$ $10^{\circ}$. This is shown graphically in the polar plot in Figure 5. The effect of the detuning is to move the response vector around the circle in the range shown.

\section{SPECIFIC CAVITY PARAMETERS}

The pertinent parameters of the cavity with the least excess margin (cavity 70) are shown in Table 1. In addition the equivalent parameters of the low beta cavity (module 19) with the least excess margin are given. Since the lower beta modules can get more excess margin by raising the power supply setting, the consideration focuses on module 70 , in which the klystron is operated at its maximum ratings, but the excess power is the minimum specified amount.

Table 1: Parameters of two cavities with minimal excess power margin

\begin{tabular}{|c|c|c|}
\hline Cavity Number & 19 & 70 \\
\hline Beam Power (kW) & 228 & 367 \\
\hline Synchronous Phase (deg) & -25.6 & -19.5 \\
\hline E-acc (MV/m) & 11.4 & 15.8 \\
\hline $\begin{array}{c}\text { Lorentz constant } \\
\left(\mathrm{Hz} /(\mathrm{MV} / \mathrm{m})^{2}\right)\end{array}$ & -2.9 & -1.8 \\
\hline Loaded Q & $7.32 \mathrm{e} 5$ & $6.98 \mathrm{e} 5$ \\
\hline Bandwidth $(\mathrm{Hz})$ & 549 & 576 \\
\hline
\end{tabular}

\section{MODELING RESULTS}

Extensive modeling has been used to determine where excess margin is needed and used [5]. The modeling considers linear/non-linear elements and time and freauency domains.

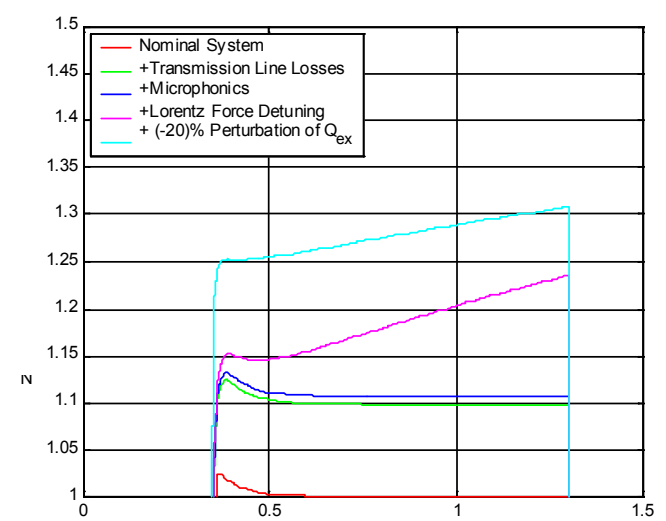

Figure 6. Excess power needed during the pulse to cumulatively accommodate the expected disturbances.

Due to mechanical time constants, Lorentz force detuning does not reach its maximum within the RF pulse. The maximum frequency excursion due to Lorentz forces is $-452 \mathrm{~Hz}$, but $-305 \mathrm{~Hz}$ is reached by the end of the RF pulse. Figure 6 shows the excess power needed to accommodate no disturbance (perfect system with beam), transmission losses and mismatches, microphonics, and Lorentz detuning. $23.5 \%$ of the allowed $40 \%$ maximum is needed. When we add in the coupler setting errors $(20 \%$ error), the excess power needed grows to $31 \%$.

We then added a white-noise, Gaussian distribution to the system in 2 places: before the feedback controller to simulate noise in RF components $(0.34 \%$ amplitude and 0.19 degrees of phase) and between the controller and the $\mathrm{RF}$ amplifier to simulate noise in the I/Q modulator, couplers, switches $(3.61 \%$ and 4.12 degrees of phase, primarily from the I/Q modulator). These levels are more than likely higher than they will actually be. In addition, $1.2 \%, 1.2^{\circ}$ noise was added to the beam, and ripple was put on the HV power supply. The amplitude and phase of the cavity with the noise inputs, all of the disturbances shown in Figure 6, and full feedback control implemented remain within the specified limits of $\pm 0.5 \%, \pm 0.5^{\circ}$ (figures 7 and 8 ).
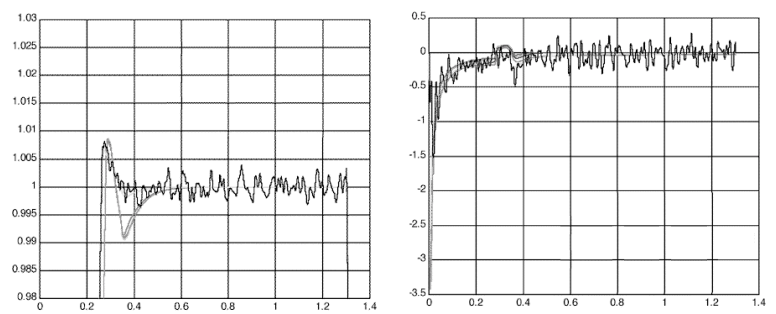

Figures 7 and 8. Amplitude and Phase of cavity with all known disturbances included. Results are within specifications $\left( \pm 0.5 \%, \pm 0.5^{\circ}\right)$.

The power margin needed to accommodate all errors, including noise, is on the order of $45 \%$. If we predetune for Lorentz forces such that optimal detuning occurs in the middle of the Lorentz swing, and if we adjust the coupler settings such that the error range of $\pm 20 \%$ is centered on the overcoupled side, since the effects of cavity coupler errors are more severe if undercoupled rather than overcoupled, the maximum excursion stays within the allowed $40 \%$ maximum.

\section{REFERENCES}

[1] J. Stovall, "Expected Beam Performance of the SNS Linac," PAC '01, Chicago, June 2001.

[2] D. Rees, et al., "The RF System Design for the Spallation Neutron Source", PAC'01, Chicago, June 2001

[3] A. Regan, et al., "The SNS Linac RF Control System", PAC'01, Chicago, June 2001.

[4] Y. Wang, et al., "System Identification of the Linac RF System Using a Wavelet Method and its Applications in the SNS LLRF Control System", PAC'01, Chicago, June 2001.

[5] S. Kwon, et al., "Uncertain System Modeling of SNS RF Control System", PAC'01, Chicago, June 2001. 